\title{
A LOCAL-TO-GLOBAL BOUNDEDNESS ARGUMENT AND FOURIER INTEGRAL OPERATORS
}

\author{
MICHAEL RUZHANSKY AND MITSURU SUGIMOTO
}

Dedicated to the memory of Professor Hans Duistermaat (1942-2010)

\begin{abstract}
We give a criterion for the global boundedness of integral operators which are known to be locally bounded. As an application, we discuss the global $L^{p}$-boundedness for a class of Fourier integral operators. While the local $L^{p_{-}}$ boundedness of Fourier integral operators is known from the work of Seeger, Sogge and Stein [SSS91, not so many results are available for the global boundedness on $L^{p}\left(\mathbb{R}^{n}\right)$. We give several natural sufficient conditions for them.
\end{abstract}

\section{INTRODUCTION}

Let $\mathcal{K}$ be an integral operator of the form

$$
\mathcal{K} u(x)=\int_{\mathbb{R}^{n}} K(x, y, x-y) u(y) d y \quad\left(x \in \mathbb{R}^{n}\right)
$$

with the kernel $K(x, y, z)$ on $\mathbb{R}_{x}^{n} \times \mathbb{R}_{y}^{n} \times \mathbb{R}_{z}^{n}$. When $K(x, y, z)=K_{0}(z)$, it is just the operator of convolution with $K_{0}$. But if we localise it by multiplication by a function $\chi$ then we immediately have such a generalised form with $K(x, y, z)=\chi(x) \chi(y) K_{0}(z)$. A more interesting example is the case when $K(x, y, z)$ is an oscillatory integral, and then $\mathcal{K}$ is called a Fourier integral operator.

Our primary objective is to give a criterion for the global boundedness

$$
\mathcal{K}: L^{p}\left(\mathbb{R}^{n}\right) \rightarrow L^{p}\left(\mathbb{R}^{n}\right), \quad 1<p<\infty,
$$

when we only know the local boundedness

$$
\mathcal{K}: L_{\text {comp }}^{p}\left(\mathbb{R}^{n}\right) \rightarrow L_{\text {loc }}^{p}\left(\mathbb{R}^{n}\right), \quad 1<p<\infty,
$$

or its endpoint boundedness

$$
\mathcal{K}: H_{\text {comp }}^{1}\left(\mathbb{R}^{n}\right) \rightarrow L_{\text {loc }}^{1}\left(\mathbb{R}^{n}\right) .
$$

Here and everywhere $H^{1}=H^{1}\left(\mathbb{R}^{n}\right)$ denotes the Hardy space introduced by Fefferman and Stein [FS72]. Let us collectively call such discussion a local-to-global boundedness argument. For example, if we further know the global $L^{2}$-boundedness and that the endpoint local boundedness is uniform with respect to the translation of localised

Date: November 6, 2018.

2010 Mathematics Subject Classification. Primary 47B38; Secondary 35S30.

Key words and phrases. Integral operators, $L^{p}$-boundedness, Fourier integral operators.

The first author was supported in parts by the EPSRC grants EP/K039407/1 and EP/R003025/1, and by the Leverhulme Grant RPG-2017-151. The second author was supported in parts by the JSPS KAKENHI 26287022 and 26610021. 
regions, then our main result (Theorem 2.2) states that we also have the global $L^{p_{-}}$ boundedness if the kernel $K(x, y, z)$ is finely controlled on a set away from its singular support. We will give the precise statement and its proof in Section 2 .

As an important application of such local-to-global boundedness argument, we will discuss the global $L^{p}$-boundedness of the Fourier integral operators

$$
\mathcal{P} u(x)=\int_{\mathbb{R}^{n}} \int_{\mathbb{R}^{n}} e^{i \phi(x, y, \xi)} a(x, y, \xi) u(y) d y d \xi \quad\left(x \in \mathbb{R}^{n}\right) .
$$

Here $\phi(x, y, \xi)$ is a real-valued function that is called a phase function while $a(x, y, \xi)$ is called an amplitude function. Following the theory of Fourier integral operators by Hörmander [Hör71, we originally assume that $\phi(x, y, \xi)$ is positively homogeneous of order 1 and smooth at $\xi \neq 0$, and that $a(x, y, \xi)$ is smooth and satisfies a growth condition in $\xi$ with some $\kappa \in \mathbb{R}$ :

$$
\sup _{(x, y) \in B}\left|\partial_{x}^{\alpha} \partial_{y}^{\beta} \partial_{\xi}^{\gamma} a(x, y, \xi)\right| \leq C_{\alpha \beta \gamma}^{B}\langle\xi\rangle^{\kappa-|\gamma|} \quad(\forall \alpha, \beta, \gamma) ; \quad\langle\xi\rangle=\left(1+|\xi|^{2}\right)^{1 / 2}
$$

for any compact set $B \subset \mathbb{R}^{n} \times \mathbb{R}^{n}$. Then the operator $\mathcal{P}$ is just a microlocal expression of the corresponding Lagrangian manifold, and with the local graph condition, it is microlocally equivalent to the special form

$$
P u(x)=\int_{\mathbb{R}^{n}} \int_{\mathbb{R}^{n}} e^{i(x \cdot \xi-\varphi(y, \xi))} a(x, y, \xi) u(y) d y d \xi
$$

by an appropriate microlocal change of variables, with possibly another amplitude.

The local $L^{p}$ mapping properties of Fourier integral operators have been extensively studied, and can be generally summarised as follows:

- $\mathcal{P}$ is $L_{c o m p}^{2}-L_{l o c}^{2}$-bounded when $\kappa \leq 0$ (Hörmander [Hör71], Eskin [Esk70]);

- $\mathcal{P}$ is $L_{\text {comp }}^{p}-L_{\text {loc }}^{p}$-bounded when $\kappa \leq-(n-1)|1 / p-1 / 2|, 1<p<\infty$ (Seeger, Sogge and Stein [SSS91]);

- $\mathcal{P}$ is $H_{\text {comp }}^{1}-L_{\text {loc }}^{1}$-bounded when $\kappa \leq-\frac{n-1}{2}$ (Seeger, Sogge and Stein [SSS91]);

- $\mathcal{P}$ is locally weak $(1,1)$ type when $\kappa \leq-\frac{n-1}{2}$ (Tao [Tao04]).

The sharpness of the order $-(n-1)|1 / p-1 / 2|$ was shown by Miyachi [Miy80] and Peral Per80] (see also SSS91]). Therefore, the question also addressed in this paper is when Fourier integral operators are globally $L^{p}$-bounded. Although the operator $\mathcal{P}$ or $P$ is just a microlocal expression of the corresponding Lagrangian manifold due to the Maslov cohomology class (see e.g. Duistermaat [Dui96]), we still regard it as a globally defined operator since it is still important for the applications to the theory of partial differential equations. Indeed, the operator $P$ is used to:

- express solutions to Cauchy problems of hyperbolic equations;

- transform operators/equations to other simpler ones (Egorov's theorem).

The typical two types of phase functions for each analysis above are

$$
\text { (I) } \varphi(y, \xi)=y \cdot \xi+|\psi(\xi)|,
$$

$$
\varphi(y, \xi)=y \cdot \psi(\xi)
$$

where $\psi(\xi)$ is a real vector-valued smooth function which is positively homogeneous of order 1 for large $\xi$. (See Definition 3.3 for the precise meaning of this terminology).

As for the global $L^{2}$-boundedness of Fourier integral operators, the following result by Asada and Fujiwara AF78] is fundamental: 
Theorem 1.1 ([AF78]). Let $\phi(x, y, \xi)$ and $a(x, y, \xi)$ be $C^{\infty}$-functions, and let

$$
D(\phi):=\left(\begin{array}{ll}
\partial_{x} \partial_{y} \phi & \partial_{x} \partial_{\xi} \phi \\
\partial_{\xi} \partial_{y} \phi & \partial_{\xi} \partial_{\xi} \phi
\end{array}\right) .
$$

Assume that $|\operatorname{det} D(\phi)| \geq C>0$. Also assume that every entry of the matrix $D(\phi)$, $a(x, y, \xi)$ and all their derivatives are bounded. Then operator $\mathcal{P}$ defined by (1.1) is $L^{2}\left(\mathbb{R}^{n}\right)$-bounded.

The result of AF78 was used to construct the solution to the Cauchy problem of Schrödinger equations by means of the Feynman path integrals in Fujiwara [Fuj79. For the operator $P$ defined by (1.2), the conditions of Theorem 1.1 are reduced to a global version of the local graph condition

$$
\left|\operatorname{det} \partial_{y} \partial_{\xi} \varphi(y, \xi)\right| \geq C>0
$$

and the growth conditions

$$
\begin{aligned}
& \left|\partial_{y}^{\alpha} \partial_{\xi}^{\beta} \varphi(y, \xi)\right| \leq C_{\alpha \beta} \quad(\forall|\alpha+\beta| \geq 2,|\beta| \geq 1), \\
& \left|\partial_{x}^{\alpha} \partial_{y}^{\beta} \partial_{\xi}^{\gamma} a(x, y, \xi)\right| \leq C_{\alpha \beta \gamma} \quad(\forall \alpha, \beta, \gamma),
\end{aligned}
$$

for all $x, y, \xi \in \mathbb{R}^{n}$. Note that the local graph condition is required even for the local $L^{2}$-boundedness of Fourier integral operators of order zero, so it is rather natural to assume (1.3) for the global $L^{2}$-boundedness. We also note that the phase functions of the type (I) satisfy the growth condition (1.4), but the type (II) does not. We mention that other types of growth conditions were introduced by the authors in RS06a] to obtain the global $L^{2}$-boundedness for operators with phase functions of the type (II), and such result was then used to show global smoothing estimates for dispersive equations in a series of papers [RS06b], RS12b] and [RS12a].

As for the global $L^{p}$-boundedness, it is deduced by our local-to-global argument from the global $L^{2}$-boundedness (Theorem 1.1) and the local endpoint result given by Seeger, Sogge and Stein [SSS91]. Indeed, in this paper we establish the following generalised result:

Theorem 1.2. Let $\varphi(y, \xi)$ and $a(x, y, \xi)$ be $C^{\infty}$-functions. Assume that $\varphi(y, \xi)$ is positively homogeneous of order 1 for large $\xi$ and satisfies (1.3). Also assume that

$$
\begin{aligned}
& \left|\partial_{y}^{\alpha} \partial_{\xi}^{\beta}(y \cdot \xi-\varphi(y, \xi))\right| \leq C_{\alpha \beta}\langle\xi\rangle^{1-|\beta|} \quad(\forall \alpha,|\beta| \geq 1), \\
& \left|\partial_{x}^{\alpha} \partial_{y}^{\beta} \partial_{\xi}^{\gamma} a(x, y, \xi)\right| \leq C_{\alpha \beta \gamma}\langle\xi\rangle^{-(n-1)|1 / p-1 / 2|-|\gamma|} \quad(\forall \alpha, \beta, \gamma),
\end{aligned}
$$

hold for all $x, y, \xi \in \mathbb{R}^{n}$. Then operator $P$ defined by (1.2) is $L^{p}\left(\mathbb{R}^{n}\right)$-bounded, for every $1<p<\infty$.

Theorem 1.2 together with some related results will be restated in Section 3 in a different form (in particular, Theorem 1.2 follows from Corollary 3.4), emphasising that they are given as an application of our local-to-global argument discussed in Section 2. We remark that Theorem 1.2 with $p=2$ was also given by Kumano-go $\operatorname{Kg} 76$. For the special cases $\varphi(y, \xi)=\varphi(\xi)$ and $a(x, y, \xi)=a(\xi)$, Theorem 1.2 was given by Miyachi [Miy80] under the assumptions that $\varphi>0$ and that the compact hypersurface

$$
\Sigma=\left\{\xi \in \mathbb{R}^{n} \backslash 0: \varphi(\xi)=1\right\}
$$


has non-zero Gaussian curvature. Beals [Bea82] and Sugimoto [Sug92] discussed the case when $\Sigma$ might have vanishing Gaussian curvature but is still convex.

We also mention that phase functions of the type (I) again satisfy the assumption of Theorem 1.2, but type (II) does not. Unfortunately our local-to-global argument does not work for the type (II) and the linear growth in $y$ causes an extra requirement for the growth order of amplitude functions. This is rather natural since one knows that in general, in the type (II) case, there is a loss in weight in global $L^{p}$ estimates for $p \neq 2$, see Coriasco and Ruzhansky [CR10, CR14 for global bounds for Fourier integral operators in this case.

To complement some references on the local and global boundedness properties of Fourier integral operators, we refer to the authors' paper [RS11] for the weighted $L^{2}$ and to Dos Santos Ferreira and Staubach [DSFS14 for other weighted properties of Fourier integral operators, to Rodríguez-López and Staubach RLS13 for estimates for rough Fourier integral operators, to Ruz01 for $L^{p}$-estimates for Fourier integral operators with complex phase functions, as well as to [Ruz09] for an earlier overview of local and global properties of Fourier integral operators with real and complex phase functions. The $L^{p}$-boundedness of bilinear Fourier integral operators has been also investigated, see e.g. Hong, Lu, Zhang [HLZ15] and references therein.

\section{A LOCAL-TO-GLOBAL BOUNDEDNESS ARGUMENT}

We discuss when the local boundedness of an integral operator induces the global one. Let $\mathcal{K}$ be an integral operator of the form

$$
\mathcal{K} u(x)=\int_{\mathbb{R}^{n}} K(x, y, x-y) u(y) d y \quad\left(x \in \mathbb{R}^{n}\right)
$$

with a measurable function $K(x, y, z)$ on $\mathbb{R}_{x}^{n} \times \mathbb{R}_{y}^{n} \times \mathbb{R}_{z}^{n}$. The formal adjoint $\mathcal{K}^{*}$ of $\mathcal{K}$ is given by

$$
\mathcal{K}^{*} u(x)=\int_{\mathbb{R}^{n}} K^{*}(x, y, x-y) u(y) d y, \quad K^{*}(x, y, z)=\overline{K(y, x,-z)} .
$$

We introduce a notion of the local boundedness. By $\chi_{B}$ we denote the multiplication by the smooth characteristic function of the set $B \subset \mathbb{R}^{n}$. As before, $H^{1}\left(\mathbb{R}^{n}\right)$ denotes the Hardy space introduced by Fefferman-Stein [FS72].

Definition 2.1. We say that the operator $\mathcal{K}$ is $H_{\text {comp }}^{1}\left(\mathbb{R}^{n}\right)-L_{\text {loc }}^{1}\left(\mathbb{R}^{n}\right)$-bounded if the localised operator $\chi_{B} \mathcal{K} \chi_{B}$ is $H^{1}\left(\mathbb{R}^{n}\right)-L^{1}\left(\mathbb{R}^{n}\right)$-bounded for any compact set $B \subset \mathbb{R}^{n}$. Furthermore, if the operator norm of $\chi_{B_{h}} \mathcal{K}_{\chi_{B_{h}}}$ is bounded in $h \in \mathbb{R}^{n}$ for the translated set $B_{h}=\{x+h: x \in B\}$ of any compact set $B \subset \mathbb{R}^{n}$, i.e. if

$$
\sup _{h \in \mathbb{R}^{n}}\left\|\chi_{B_{h}} \mathcal{K} \chi_{B_{h}}\right\|_{H^{1}\left(\mathbb{R}^{n}\right) \rightarrow L^{1}\left(\mathbb{R}^{n}\right)}<\infty,
$$

we say that the operator $\mathcal{K}$ is uniformly $H_{\text {comp }}^{1}\left(\mathbb{R}^{n}\right)-L_{\text {loc }}^{1}\left(\mathbb{R}^{n}\right)$-bounded.

If we introduce the translation operator $\tau_{h}: f(x) \mapsto f(x-h)$ and its inverse (formal adjoint) $\tau_{h}^{*}=\tau_{-h}$, we have the equality $\chi_{B_{h}}=\tau_{h} \chi_{B} \tau_{h}^{*}$. Since $L^{1}$ and $H^{1}$ norms are translation invariant, $\mathcal{K}$ is uniformly $H_{c o m p}^{1}-L_{l o c}^{1}$-bounded if and only if $\chi_{B}\left(\tau_{h}^{*} \mathcal{K} \tau_{h}\right) \chi_{B}$ 
is $H^{1}$ - $L^{1}$-bounded for any compact set $B \subset \mathbb{R}^{n}$ and the operator norms are bounded in $h \in \mathbb{R}^{n}$. We remark that the operator $\tau_{h}^{*} \mathcal{K} \tau_{h}$ has the expression

$$
\tau_{h}^{*} \mathcal{K} \tau_{h} u(x)=\int_{\mathbb{R}^{n}} K_{h}(x, y, x-y) u(y) d y, \quad K_{h}(x, y, z)=K(x+h, y+h, z)
$$

We have the following main result:

Theorem 2.2. Suppose that operator $\mathcal{K}$ defined by (2.1) is $L^{2}\left(\mathbb{R}^{n}\right)$-bounded and uniformly $H_{\text {comp }}^{1}\left(\mathbb{R}^{n}\right)-L_{\text {loc }}^{1}\left(\mathbb{R}^{n}\right)$-bounded. Assume that there exits a measurable function $H(x, y, z)$ which satisfies the following condition:

(A1) There exist constants $d>0$ and $k>n$ such that

$$
\sup _{H(x, y, z) \geq d}\left|H(x, y, z)^{k} K(x, y, z)\right|<\infty \text {. }
$$

Furthermore, we set

$$
\widetilde{H}(z):=\inf _{x, y \in \mathbb{R}^{n}} H(x, y, z) .
$$

and assume also the following two conditions:

(A2) There exist constants $A>0$ and $A_{0}>0$ such that

$$
\widetilde{H}(z) \geq A_{0}|z| \text { whenever }|z| \geq A \text {. }
$$

(A3) There exist constants $b>0$ and $b_{0}>0$ such that

$$
\widetilde{H}(z) \leq b_{0} \widetilde{H}\left(z-z^{\prime}\right) \text { whenever } \widetilde{H}(z) \geq b\left|z^{\prime}\right| .
$$

Then $\mathcal{K}$ is $H^{1}\left(\mathbb{R}^{n}\right)-L^{1}\left(\mathbb{R}^{n}\right)$-bounded. If in addition operator $\mathcal{K}^{*}$ defined by (2.2) is uniformly $H_{\text {comp }}^{1}\left(\mathbb{R}^{n}\right)$ - $L_{\text {loc }}^{1}\left(\mathbb{R}^{n}\right)$-bounded, then $\mathcal{K}^{*}$ is also $H^{1}\left(\mathbb{R}^{n}\right)-L^{1}\left(\mathbb{R}^{n}\right)$-bounded.

Theorem 2.2 means that the global $L^{2}$-boundedness and some additional assumptions induce the global $H^{1}-L^{1}$-boundedness form the local one. Then, if we want, we can have the global $L^{p}$-boundedness for $1<p<\infty$ by the interpolation and the duality argument. Immediate examples to which Theorem 2.2 can be applied are pseudo-differential operators

$$
\begin{aligned}
\mathcal{P}_{s} u(x) & =\iint_{\mathbb{R}^{n} \times \mathbb{R}^{n}} e^{i(x-y) \cdot \xi} a(x, y, \xi) u(y) d y d \xi \\
& =\int_{\mathbb{R}^{n}} K(x, y, x-y) u(y) d y
\end{aligned}
$$

where

$$
K(x, y, z)=\int_{\mathbb{R}^{n}} e^{i z \cdot \xi} a(x, y, \xi) d \xi .
$$

(Indeed, we can take $H(x, y, z)=|z|$ in this case if $a(x, y, \xi)$ belongs to a standard symbol class.) More interesting examples for which Theorem 2.2 yields new $L^{p}$ boundedness results are Fourier integral operators which include pseudo-differential operators as special ones. They will be intensively discussed in the next section.

Now we give the proof of Theorem 2.2. We only show the assertion for $\mathcal{K}$ because conditions (A1)-(A3) induce corresponding conditions for the kernel $K^{*}(x, y, z)$ in (2.2) of $\mathcal{K}^{*}$ if we take $H^{*}(x, y, z)=H(y, x,-z)$. Furthermore, we may take $d=1$ in (A1) otherwise replace $H$ by $H / d$, and we may also take $b=b_{0}$ in (A3) otherwise replace the smaller one by the bigger. 
We introduce the notations

$$
\Delta_{r}:=\left\{(x, y, z) \in \mathbb{R}^{n} \times \mathbb{R}^{n} \times \mathbb{R}^{n}: H(x, y, z) \geq r\right\}
$$

and

$$
\widetilde{\Delta}_{r}:=\left\{z \in \mathbb{R}^{n}: \widetilde{H}(z) \geq r\right\} .
$$

Clearly we have the monotonicity of $\Delta_{r}$ and $\widetilde{\Delta}_{r}$ in $r>0$, that is, $\Delta_{r_{1}} \subset \Delta_{r_{2}}$, $\widetilde{\Delta}_{r_{1}} \subset \widetilde{\Delta}_{r_{2}}$ for $r_{1} \geq r_{2} \geq 0$. On account of them, we have the following:

Lemma 2.1. Let $r \geq 1$ and let $h \in \mathbb{R}^{n}$. Suppose $\operatorname{supp} f \subset\left\{x \in \mathbb{R}^{n}:|x| \leq r\right\}$. Then we have

$$
\left\|\tau_{h}^{*} \mathcal{K} \tau_{h} f\right\|_{L^{1}\left(\widetilde{\Delta}_{b r}\right)} \leq C\|f\|_{L^{1}}
$$

where $C$ is a positive constant independent of $r$ and $h$.

Proof. First we consider the case $h=0$. For $x \in \widetilde{\Delta}_{b r}$ and $|y| \leq r$, we have $\widetilde{H}(x) \geq b r$ by the definition of $\Delta_{b r}$, and hence we also have $\widetilde{H}(x) \geq b|y|$. Then from (A3) with $b=b_{0}$, we obtain

$$
b r \leq \widetilde{H}(x) \leq b \widetilde{H}(x-y) \leq b H(x, y, x-y)
$$

which implies $(x, y, x-y) \in \Delta_{r}$ and $\widetilde{H}(x, y, x-y)^{-1} \leq b \widetilde{H}(x)^{-1}$. Then we have

$$
\begin{aligned}
|\mathcal{K} f(x)| & \leq b^{k} \widetilde{H}(x)^{-k} \int_{|y| \leq r}\left|H(x, y, x-y)^{k} K(x, y, x-y) f(y)\right| d y \\
& \leq b^{k} \widetilde{H}(x)^{-k}\left\|H(x, y, z)^{k} K(x, y, z)\right\|_{L^{\infty}\left(\Delta_{r}\right)}\|f\|_{L^{1}},
\end{aligned}
$$

for $x \in \widetilde{\Delta}_{b r}$. Hence, by the monotonicity $\Delta_{b r} \subset \Delta_{b}$ and $\widetilde{\Delta}_{r} \subset \widetilde{\Delta}_{1}(r \geq 1)$, we have

$$
\begin{aligned}
\|\mathcal{K} f\|_{L^{1}\left(\widetilde{\Delta}_{b r}\right)} & \leq b^{k}\left\|\widetilde{H}(x)^{-k}\right\|_{L^{1}\left(\widetilde{\Delta}_{b r}\right)}\left\|H(x, y, z)^{k} K(x, y, z)\right\|_{L^{\infty}\left(\Delta_{r}\right)}\|f\|_{L^{1}} \\
& \leq b^{k}\left\|\widetilde{H}(x)^{-k}\right\|_{L^{1}\left(\widetilde{\Delta}_{b}\right)}\left\|H(x, y, z)^{k} K(x, y, z)\right\|_{L^{\infty}\left(\Delta_{1}\right)}\|f\|_{L^{1}} \\
& \leq C\|f\|_{L^{1}},
\end{aligned}
$$

for $k>n$, where we have used (A2) to justify the estimate

$$
\begin{aligned}
\left\|\widetilde{H}(z)^{-k}\right\|_{L^{1}\left(\widetilde{\Delta}_{b}\right)} & \leq\left\|\widetilde{H}(z)^{-k}\right\|_{L^{1}\left(\widetilde{\Delta}_{b} \cap\{|z| \leq A\}\right)}+\left\|\widetilde{H}(z)^{-k}\right\|_{L^{1}\left(\widetilde{\Delta}_{b} \cap\{|z| \geq A\}\right)} \\
& \leq b^{-k}\|1\|_{L^{1}(|z| \leq A)}+A_{0}^{-k}\left\||z|^{-k}\right\|_{L^{1}(|z| \geq A)} \\
& \leq C
\end{aligned}
$$

and also (A1) with $d=1$.

For general $h \in \mathbb{R}^{n}$, we apply the same argument for $K_{h}$ in (2.3) and

$$
H_{h}(x, y, z)=H(x-h, y-h, z)
$$

instead of $K$ and $H$, respectively. We remark that conditions (A1), (A2) and (A3) in Theorem 2.2 are invariant in $h \in \mathbb{R}^{n}$ in the sense that we have

$$
\begin{aligned}
& \sup _{H_{h}(x, y, z) \geq d}\left|H_{h}(x, y, z)^{k} K_{h}(x, y, z)\right|=\sup _{H(x, y, z) \geq d}\left|H(x, y, z)^{k} K(x, y, z)\right|, \\
& \widetilde{H_{h}}(z)=\inf _{x, y \in \mathbb{R}^{n}} H_{h}(x, y, z)=\widetilde{H}(z) .
\end{aligned}
$$


Then we have the same estimates with the same constants but $\mathcal{K}$ replaced by $\tau_{h}^{*} \mathcal{K} \tau_{h}$. This finishes the proof.

Lemma 2.2. Let $r \geq 1$. Then there exists a constant $c>0$ independent of $r$ such that $\mathbb{R}^{n} \backslash \widetilde{\Delta}_{b r} \subset\{z:|z|<c r\}$.

Proof. Let $z \in \mathbb{R}^{n} \backslash \widetilde{\Delta}_{b r}$, that is, $\widetilde{H}(z)<b r$. By the definition of $\widetilde{H}(z)$, there exist $x_{0}, y_{0}, \xi_{0} \in \mathbb{R}^{n}$ such that $H\left(x_{0}, y_{0}, z\right) \leq 2 \widetilde{H}(z)$, and by (A2) we have $A_{0}|z| \leq$ $H\left(x_{0}, y_{0}, z\right)$ for $|z| \geq A$. Hence we have $|z| \leq\left(2 b / A_{0}\right) r$ for $|z| \geq A$. On the other hand, we always have $|z| \leq A r$ for $|z| \leq A$ since $r \geq 1$, and we have the conclusion.

Now we are ready to prove the $H^{1}-L^{1}$-boundedness. We use the characterisation of $H^{1}$ by the atomic decomposition proved by Coifman and Weiss [CW77]. That is, any $f \in H^{1}\left(\mathbb{R}^{n}\right)$ can be represented as

$$
f=\sum_{j=1}^{\infty} \lambda_{j} g_{j}, \quad \lambda_{j} \in \mathbb{C}, \quad g_{j}: \text { atom }
$$

and the norm $\|f\|_{H^{1}}$ is equivalent to the norm $\left\|\left\{\lambda_{j}\right\}_{j=1}^{\infty}\right\|_{\ell^{1}}=\sum_{j=1}^{\infty}\left|\lambda_{j}\right|$. Here we call a function $g$ on $\mathbb{R}^{n}$ an atom if there is a ball $B=B_{g} \subset \mathbb{R}^{n}$ such that $\operatorname{supp} g \subset B$, $\|g\|_{L^{\infty}} \leq|B|^{-1}(|B|$ is the Lebesgue measure of the ball $B)$ and $\int g(x) d x=0$. From this, all we have to show is the estimate

$$
\|\mathcal{K} g\|_{L^{1}\left(\mathbb{R}^{n}\right)} \leq C,
$$

with some constant $C>0$ for all atoms $g$. By an appropriate translation, it is further reduced to the estimate

$$
\left\|\tau_{h}^{*} \mathcal{K} \tau_{h} f\right\|_{L^{1}\left(\mathbb{R}^{n}\right)} \leq C, \quad f \in \mathcal{A}_{r},
$$

where $\mathcal{A}_{r}$ is the set of all functions $f$ on $\mathbb{R}^{n}$ such that

$$
\text { supp } f \subset B_{r}=\left\{x \in \mathbb{R}^{n}:|x| \leq r\right\}, \quad\|f\|_{L^{\infty}} \leq\left|B_{r}\right|^{-1}, \quad \int f(x) d x=0 .
$$

Here and hereafter in this section, $C$ always denotes a constant which is independent of $h \in \mathbb{R}^{n}$ and $0<r<\infty$, and which may differ from one formula to another.

Suppose $f \in \mathcal{A}_{r}$ with $r \geq 1$. Then we split $\mathbb{R}^{n}$ into two parts $\widetilde{\Delta}_{b r}$ and $\mathbb{R}^{n} \backslash \widetilde{\Delta}_{b r}$. For the part $\widetilde{\Delta}_{b r}$, we have by Lemma 2.1 that

$$
\left\|\tau_{h}^{*} \mathcal{K} \tau_{h} f\right\|_{L^{1}\left(\widetilde{\Delta}_{b r}\right)} \leq C\|f\|_{L^{1}} \leq C
$$

For the part $\mathbb{R}^{n} \backslash \widetilde{\Delta}_{b r}$, we have by Lemma 2.2 and the Cauchy-Schwarz inequality

$$
\begin{aligned}
\left\|\tau_{h}^{*} \mathcal{K} \tau_{h} f\right\|_{L^{1}\left(\mathbb{R}^{n} \backslash \widetilde{\Delta}_{b r}\right)} & \leq\|1\|_{L^{2}(|x|<c r)}\left\|\tau_{h}^{*} \mathcal{K} \tau_{h} f\right\|_{L^{2}\left(\mathbb{R}^{n}\right)} \\
& \leq C r^{n / 2}\|f\|_{L^{2}\left(\mathbb{R}^{n}\right)} \leq C
\end{aligned}
$$

where we have used the assumption that $\mathcal{K}$ is $L^{2}$-bounded, and (2.4) in the last inequality. 
Suppose now $f \in \mathcal{A}_{r}$ with $r \leq 1$. Then we split $\mathbb{R}^{n}$ into the parts $\Delta_{b}$ and $\mathbb{R}^{n} \backslash \Delta_{b}$. For the part $\Delta_{b}$, we have by Lemma 2.1 with $r=1$ and the inclusion supp $f \subset B_{r} \subset$ $B_{1}$

$$
\left\|\tau_{h}^{*} \mathcal{K} \tau_{h} f\right\|_{L^{1}\left(\widetilde{\Delta}_{b}\right)} \leq C\|f\|_{L^{1}} \leq C .
$$

For the part $\mathbb{R}^{n} \backslash \Delta_{b}$, we have by Lemma 2.2 that

$$
\begin{aligned}
\left\|\tau_{h}^{*} \mathcal{K} \tau_{h} f\right\|_{L^{1}\left(\mathbb{R}^{n} \backslash \Delta_{b}\right)} & \leq\left\|\tau_{h}^{*} \mathcal{K} \tau_{h} f\right\|_{L^{1}(|x|<c)} \\
& \leq C\|f\|_{H^{1}} \leq C,
\end{aligned}
$$

where we have used the fact that $\mathcal{K}$ is uniformly $H_{\text {comp }}^{1} L_{\text {loc }}^{1}$-bounded. Thus the proof of Theorem 2.2 is complete.

\section{Fourier integral Operators}

A typical example of integral operators (2.1) which we have in mind is Fourier integral operators of the form

$$
\mathcal{P} u(x)=\int_{\mathbb{R}^{n}} \int_{\mathbb{R}^{n}} e^{i \phi(x, y, \xi)} a(x, y, \xi) u(y) d y d \xi \quad\left(x \in \mathbb{R}^{n}\right) .
$$

For convenience, we introduce the function $\Phi(x, y, \xi)$ to write

$$
\phi(x, y, \xi)=(x-y) \cdot \xi+\Phi(x, y, \xi)
$$

and then we have the kernel representation

$$
\mathcal{P} u(x)=\int_{\mathbb{R}^{n}} K(x, y, x-y) u(y) d y
$$

with

$$
K(x, y, z)=\int_{\mathbb{R}^{n}} e^{i\{z \cdot \xi+\Phi(x, y, \xi)\}} a(x, y, \xi) d \xi .
$$

In particular, $\mathcal{P}$ is a pseudo-differential operator when $\Phi(x, y, \xi)=0$. We remark that the formal adjoint $\mathcal{P}^{*}$ of $\mathcal{P}$ is of the same form (3.1) with the replacement

$$
\begin{aligned}
& \Phi(x, y, \xi) \longmapsto \Phi^{*}(x, y, \xi)=-\Phi(y, x, \xi), \\
& a(x, y, \xi) \longmapsto a^{*}(x, y, \xi)=\overline{a(y, x, \xi)},
\end{aligned}
$$

and also the operator $\tau_{h}^{*} \mathcal{P} \tau_{h}$ with

$$
\begin{aligned}
& \Phi(x, y, \xi) \longmapsto \Phi^{h}(x, y, \xi)=\Phi(x+h, y+h, \xi), \\
& a(x, y, \xi) \longmapsto a^{h}(x, y, \xi)=a(x+h, y+h, \xi),
\end{aligned}
$$

as special cases of the general rules (2.2) and (2.3).

We introduce a class of amplidtude functions $a(x, y, \xi)$ :

Definition 3.1. For $\kappa \in \mathbb{R}, S^{\kappa}$ denotes the class of smooth functions $a=a(x, y, \xi) \in$ $C^{\infty}\left(\mathbb{R}^{n} \times \mathbb{R}^{n} \times \mathbb{R}^{n}\right)$ satisfying the estimate

$$
\left|\partial_{x}^{\alpha} \partial_{y}^{\beta} \partial_{\xi}^{\gamma} a(x, y, \xi)\right| \leq C_{\alpha \beta \gamma}\langle\xi\rangle^{\kappa-|\gamma|}
$$

for all $x, y, \xi \in \mathbb{R}^{n}$ and all multi-indices $\alpha, \beta, \gamma$. 
Let us now try to apply Theorem 2.2 from the previous section for Fourier integral operators defined by (3.1). Our natural choice of $H(x, y, z)$ is a defining function of the singular support of the kernel. For example, the kernels of pseudo-differential operators (that is, (3.3) with $\Phi(x, y, z)=0$ ) is singular only when $z=0$, and we can take $H(x, y, z)=|z|$. Indeed we can easily see that it satisfies assumptions (A1)-(A3) in Theorem 2.2 if $a(x, y, \xi)$ belongs to a class $S^{m}$. For general Fourier integral operators, we can find $H(x, y, z)$ corresponding to $\Phi(x, y, z)$ by the same consideration:

Lemma 3.1. Assume that $a=a(x, y, \xi) \in S^{\kappa}$ with some $\kappa \in \mathbb{R}$, and assume also that $\Phi(x, y, \xi)$ is a real-valued $C^{\infty}$-function and that $\partial_{\xi}^{\gamma} \Phi(x, y, \xi) \in S^{0}$ for $|\gamma|=1$. Let

$$
H(x, y, z):=\inf _{\xi \in \mathbb{R}^{n}}\left|z+\nabla_{\xi} \Phi(x, y, \xi)\right|,
$$

and let

$$
\widetilde{H}(z):=\inf _{x, y \in \mathbb{R}^{n}} H(x, y, z)=\inf _{x, y, \xi \in \mathbb{R}^{n}}\left|z+\nabla_{\xi} \Phi(x, y, \xi)\right| .
$$

Then $K(x, y, z)$ defined by (3.3) satisfies assumptions (A1)-(A3) in Theorem 2.2.

Proof. The expression (3.3) is justified by the integration by parts

$$
K(x, y, z)=\int_{\mathbb{R}^{n}} e^{i\{z \cdot \xi+\Phi(x, y, \xi)\}}\left(L^{*}\right)^{n+1} a(x, y, \xi) d \xi
$$

outside the set

$$
\begin{aligned}
\Sigma & =\left\{\left(x, y,-\nabla_{\xi} \Phi(x, y, \xi)\right) \in \mathbb{R}^{n} \times \mathbb{R}^{n} \times \mathbb{R}^{n}: x, y, \xi \in \mathbb{R}^{n}\right\} \\
& =\left\{(x, y, z) \in \mathbb{R}^{n} \times \mathbb{R}^{n} \times \mathbb{R}^{n}: H(x, y, z)=0\right\},
\end{aligned}
$$

where $L^{*}$ is the transpose of the operator

$$
L=\frac{\left(z+\nabla_{\xi} \Phi\right) \cdot \nabla_{\xi}}{i\left|z+\nabla_{\xi} \Phi\right|^{2}}
$$

Noticing that $d \leq H(x, y, z)$ implies $d \leq\left|z+\nabla_{\xi} \Phi(x, y, \xi)\right|$ for all $\xi \in \mathbb{R}^{n}$, we easily have (A1). On the other hand, we have

$$
|z| \leq\left|z+\nabla_{\xi} \Phi(x, y, \xi)\right|+\left|\nabla_{\xi} \Phi(x, y, \xi)\right| \leq\left|z+\nabla_{\xi} \Phi(x, y, \xi)\right|+N,
$$

with some constant $N>0$ for any $x, y, \xi \in \mathbb{R}^{n}$, hence $|z| \leq \widetilde{H}(z)+N$. Then for $|z| \geq 2 N$ we have $|z| \leq \widetilde{H}(z)+|z| / 2$, hence $\widetilde{H}(z) \geq|z| / 2$, that is, (A2). Finally, if $\widetilde{H}(z) \geq 2\left|z^{\prime}\right|$, then we have

$$
\begin{aligned}
\widetilde{H}(z) & \leq H(x, y, z) \leq\left|z+\nabla_{\xi} \Phi(x, y, \xi)\right| \leq\left|z-z^{\prime}+\nabla_{\xi} \Phi(x, y, \xi)\right|+\left|z^{\prime}\right| \\
& \leq\left|z-z^{\prime}+\nabla_{\xi} \Phi(x, y, \xi)\right|+\widetilde{H}(z) / 2
\end{aligned}
$$

hence $\widetilde{H}(z) \leq 2\left|z-z^{\prime}+\nabla_{\xi} \Phi(x, y, \xi)\right|$ for all $x, y, \xi \in \mathbb{R}^{n}$, hence $\widetilde{H}(z) \leq 2 \widetilde{H}\left(z-z^{\prime}\right)$ that is, we have (A3).

From Lemma 3.1, we immediately obtain the following result from Theorem 2.2.

Theorem 3.2. Let $\mathcal{P}$ be a operator defined by (3.1) with (3.2). Let $1<p<\infty$, let $\kappa_{1} \leq 0$, and let $\kappa \leq 2 \kappa_{1}|1 / p-1 / 2|$ Assume the following conditions:

(B1) $\Phi(x, y, \xi)$ is a real-valued $C^{\infty}$-function and $\partial_{\xi}^{\gamma} \Phi(x, y, \xi) \in S^{0}$ for $|\gamma|=1$. 
(B2) $\mathcal{P}$ is $L^{2}\left(\mathbb{R}^{n}\right)$-bounded whenever $a(x, y, \xi) \in S^{0}$.

(B3) $\mathcal{P}$ and $\mathcal{P}^{*}$ are uniformly $H_{\text {comp }}^{1}\left(\mathbb{R}^{n}\right)-L_{\text {loc }}^{1}\left(\mathbb{R}^{n}\right)$-bounded whenever $a=a(x, y, \xi) \in$ $S^{\kappa_{1}}$.

Then $\mathcal{P}$ is $L^{p}\left(\mathbb{R}^{n}\right)$-bounded for any $a=a(x, y, \xi) \in S^{\kappa}$.

Indeed, by Theorem 2.2 and Lemma 3.1, assumptions (B1)-(B3) induce the $H^{1}$ $L^{1}$-boundedness of the operators $\mathcal{P}$ and $\mathcal{P}^{*}$ for $a=a(x, y, \xi) \in S^{\kappa_{1}}$ if we notice that $S^{\kappa_{1}} \subset S^{0}$. Then by the duality and the complex interpolation argument, we have the $L^{p}$-boundedness of $\mathcal{P}$ with the critical case $a=a(x, y, \xi) \in S^{2 \kappa_{1}|1 / p-1 / 2|}$, hence also for $a=a(x, y, \xi) \in S^{\kappa}$ since $S^{\kappa} \subset S^{2 \kappa_{1}|1 / p-1 / 2|}$. We remark that we can also conclude the $L^{\infty}-B M O$-boundedness of $\mathcal{P}$ if $a=a(x, y, \xi) \in S^{\kappa_{1}}$.

Assumptions (B2) and (B3) in Theorem 3.2 are essentially the requirements for phase functions $\Phi(x, y, \xi)$. A condition for (B2) is given by Asada and Fujiwara [AF78, while (B3) is given by Seeger, Sogge and Stein [SSS91]. We state further conclusions based on them by restricting our phase functions to the form

$$
\phi(x, y, \xi)=x \cdot \xi-\varphi(y, \xi) \quad(\text { in other words } \Phi(x, y, \xi)=y \cdot \xi-\varphi(y, \xi)) .
$$

First we make precise the notion of homogeneity:

Definition 3.3. We say that $\varphi=\varphi(y, \xi)$ is positively homogeneous of order 1 if

$$
\varphi(y, \lambda \xi)=\lambda \varphi(y, \xi)
$$

holds for all $y \in \mathbb{R}^{n}, \xi \neq 0$ and $\lambda>0$. We also say that $\varphi=\varphi(y, \xi)$ is positively homogeneous of order 1 for large $\xi$ if there exist a constant $R>0$ such that (3.6) holds for all $y \in \mathbb{R}^{n},|\xi| \geq R$ and $\lambda \geq 1$.

For the operator of the form

$$
P u(x)=\int_{\mathbb{R}^{n}} \int_{\mathbb{R}^{n}} e^{i(x \cdot \xi-\varphi(y, \xi))} a(x, y, \xi) u(y) d y d \xi \quad\left(x \in \mathbb{R}^{n}\right),
$$

we have the following boundedness:

Corollary 3.4. Let $1<p<\infty$ and let $\kappa \leq-(n-1)|1 / p-1 / 2|$. Assume that $a=a(x, y, \xi) \in S^{\kappa}$. Assume also the following conditions:

(C1) $\varphi(y, \xi)$ is a real-valued $C^{\infty}$-function and $\partial_{\xi}^{\gamma}(y \cdot \xi-\varphi(y, \xi)) \in S^{0}$ for $|\gamma|=1$.

(C2) There exists a constant $C>0$ such that $\left|\operatorname{det} \partial_{y} \partial_{\xi} \varphi(y, \xi)\right| \geq C$ for all $y, \xi \in \mathbb{R}^{n}$.

(C3) $\varphi(y, \xi)$ is positively homogeneous of order 1 for large $\xi$.

Then operator $P$ defined by (3.7) is $L^{p}\left(\mathbb{R}^{n}\right)$-bounded.

Proof. Let us induce assumptions (B1)-(B3) of Theorem 3.2 with $\kappa_{1}=-(n-1) / 2$ from the assumptions (C1)-(C3) of Corollary 3.4 for the special case $\phi(x, y, \xi)=$ $x \cdot \xi-\varphi(y, \xi)$ or, in other words, for $\Phi(x, y, \xi)=y \cdot \xi-\varphi(y, \xi)$. We remark that (B1) is just an interpretation of assumption (C1). As for (B2), a sufficient condition for the $L^{2}$-boundededness of $\mathcal{P}$ is known from Asada and Fujiwara [AF78, that is, Theorem 1.1 in Introduction. In particular, (B2) is fulfilled if $(\mathrm{C} 1)$ and $(\mathrm{C} 2)$ are satisfied.

Let us discuss (B3). A sufficient condition for the $H_{c o m p}^{1}-L_{l o c}^{1}$-boundedness of $P$ is known by the work of Seeger, Sogge and Stein [SSS91], that is, $P$ is $H_{c o m p}^{1} L_{l o c}^{1}$ bounded for $a=a(x, y, \xi) \in S^{-(n-1) / 2}$ if $\varphi(y, \xi)$ is a real-valued $C^{\infty}$-function on $\mathbb{R}^{n} \times\left(\mathbb{R}^{n} \backslash 0\right)$ and positively homogeneous of order 1 . If we carefully trace the 
argument in [SSS91], we can say that $\chi_{K} P \chi_{K}$ is $H^{1}\left(\mathbb{R}^{n}\right)-L^{1}\left(\mathbb{R}^{n}\right)$-bounded for any compact set $K \subset \mathbb{R}^{n}$ and its operator norm is bounded by a constant depending only on $n, K$ and quantities

$$
\begin{aligned}
& M_{\ell}=\sum_{|\alpha|+|\beta|+|\gamma| \leq \ell} \sup _{x, y, \xi \in \mathbb{R}^{n}}\left|\partial_{x}^{\alpha} \partial_{y}^{\beta} \partial_{\xi}^{\gamma} a(x, y, \xi)\langle\xi\rangle^{(n-1) / 2+|\gamma|)}\right|, \\
& N_{\ell}=\left.\sum_{\substack{|\beta| \leq \ell \\
1 \leq|\gamma| \leq \ell}} \sup _{\substack{x, y \in \mathbb{R}^{n} \\
\xi \neq 0}}\left|\partial_{y}^{\beta} \partial_{\xi}^{\gamma}(y \cdot \xi-\varphi(y, \xi))\right| \xi\right|^{-(1-|\gamma|)} \mid,
\end{aligned}
$$

with some large $\ell$. The same is true for $P^{*}$ if we trace the argument in SSte93. instead but we require $(\mathrm{C} 2)$ in this case. Then $P$ and $P^{*}$ are uniformly $H_{c o m p}^{1} L_{l o c}^{1}$ bounded if $M_{\ell}$ and $N_{\ell}$ are finite since the quantities $M_{\ell}$ and $N_{\ell}$ are invariant under the replacements (3.4) and (3.5).

Based on this fact, $P$ and $P^{*}$ are uniformly $H_{c o m p}^{1} L_{l o c}^{1}$-bounded if $a \in S^{-(n-1) / 2}$ under the assumptions $(\mathrm{C} 1)-(\mathrm{C} 3)$. In fact, let us split $a(x, y, \xi)$ into the sum of $a(x, y, \xi) g(\xi)$ and $a(x, y, \xi)(1-g(\xi))$ with an appropriate smooth cut-off function $g \in C_{0}^{\infty}\left(\mathbb{R}^{n}\right)$ which is equal to 1 near the origin. Then for the terms $P_{1}$ and $P_{1}^{*}$ corresponding to $a(x, y, \xi)(1-g(\xi))$, we can regard $\varphi(y, \xi)$ as a positively homogeneous function of order 1 by a modification near $\xi=0$, and they are uniformly $H_{\text {comp }}^{1}$ $L_{l o c}^{1}$-bounded by the above observation. On the other hand, the terms $P_{2}$ and $P_{2}^{*}$ corresponding to $a(x, y, \xi) g(\xi)$ are $L^{1}$-bounded (hence uniformly $H_{c o m p}^{1}-L_{l o c}^{1}$-bounded) because

$$
\begin{aligned}
& P_{2} u(x)=\int K(x, y) u(y) d y, \quad P_{2}^{*} u(x)=\int \overline{K(y, x)} u(y) d y, \\
& K(x, y)=\int_{\mathbb{R}^{n}} e^{i(x \cdot \xi-\phi(y, \xi))} a(x, y, \xi) g(\xi) d \xi
\end{aligned}
$$

and the integral kernel $K(x, y)$ is integrable in both $x$ and $y$. This fact can be verified by the integration by parts

$$
\begin{aligned}
K(x, y) & =\left(1+|x-y|^{2}\right)^{-n} \int_{\mathbb{R}^{n}}\left(1-\Delta_{\xi}\right)^{n} e^{i(x-y) \cdot \xi} \cdot e^{i(y \cdot \xi-\phi(y, \xi))} a(x, y, \xi) g(\xi) d \xi \\
& =\left(1+|x-y|^{2}\right)^{-n} \int_{\mathbb{R}^{n}} e^{i(x-y) \cdot \xi} \cdot\left(1-\Delta_{\xi}\right)^{n}\left\{e^{i(y \cdot \xi-\phi(y, \xi))} a(x, y, \xi) g(\xi)\right\} d \xi
\end{aligned}
$$

followed by the the conclusion

$$
|K(x, y)| \leq C\left(1+|x-y|^{2}\right)^{-n}
$$

because of assumptions (C1), $a \in S^{-(n-1) / 2}$, and $g \in C_{0}^{\infty}$.

As a conclusion, (B3) is fulfilled if (C1)-(C3) are satisfied, and the proof of Corollary 3.4 is complete.

We can admit positively homogeneous phase functions which might have singularity at the origin for a special kind of operators of the form

$$
T u(x)=\int_{\mathbb{R}^{n}} e^{i(x \cdot \xi+\psi(\xi))} a(x, \xi) \widehat{u}(\xi) d \xi \quad\left(x \in \mathbb{R}^{n}\right) .
$$

For such operators we have the following boundedness: 
Corollary 3.5. Let $1<p<\infty$ and let $\kappa \leq-(n-1)|1 / p-1 / 2|$. Assume that $a=a(x, \xi) \in S^{\kappa}$ and that $\psi=\psi(\xi)$ is a real-valued $C^{\infty}$-function on $\mathbb{R}^{n} \backslash 0$ which is positively homogeneous of order 1 . Then the operator $T$ defined by (3.8) is $L^{p}\left(\mathbb{R}^{n}\right)$ bounded.

Proof. Again we spilt the amplitude $a(x, \xi)$ into the sum of $a(x, \xi) g(\xi)$ and $a(x, \xi)(1-$ $g(\xi))$ as in the proof of Corollary 3.4. We remark that the operator $T$ defined by (3.8) is the operator $P$ defined by (3.7) with $\varphi(y, \xi)=y \cdot \xi-\psi(\xi)$ and $a(x, y, \xi)=a(x, \xi)$ independent of $y$. For the term $T_{1}$ corresponding to $a(x, \xi)(1-g(\xi))$, we just apply Corollary 3.4. For the term $T_{2}$ corresponding to $a(x, \xi) g(\xi)$, we have

$$
T_{2} u(x)=\int_{\mathbb{R}^{n}} e^{i(x \cdot \xi+\psi(\xi))} a(x, \xi) g(\xi) \widehat{u}(\xi) d \xi=a\left(X, D_{x}\right) e^{i \psi\left(D_{x}\right)} g\left(D_{x}\right) u(x) .
$$

The pseudo-differential operator $a\left(X, D_{x}\right)$ is $L^{p}$-bounded (see Kumano-go and Nagase $[\mathrm{KgN70})$ and the Fourier multiplier $e^{i \psi\left(D_{x}\right)} g\left(D_{x}\right)$ is also $L^{p}$-bounded by the Marcinkiewicz theorem (see Stein [Ste70]) since $\left|\partial^{\alpha}\left(e^{i \psi(\xi)} g(\xi)\right)\right| \leq C_{\alpha}|\xi|^{-|\alpha|}$ for any multi-index $\alpha$. The proof of Corollary 3.5 is complete.

\section{REFERENCES}

[AF78] K. Asada and D. Fujiwara. On some oscillatory integral transformations in $L^{2}\left(\mathbf{R}^{n}\right)$. Japan. J. Math. (N.S.), 4(2):299-361, 1978.

[Bea82] M. Beals. $L^{p}$ boundedness of Fourier integral operators. Mem. Amer. Math. Soc., 38(264):viii+57, 1982.

[CR10] S. Coriasco and M. Ruzhansky. On the boundedness of Fourier integral operators on $L^{p}\left(\mathbb{R}^{n}\right)$. C. R. Math. Acad. Sci. Paris, 348(15-16):847-851, 2010.

[CR14] S. Coriasco and M. Ruzhansky. Global $L^{p}$ continuity of Fourier integral operators. Trans. Amer. Math. Soc., 366(5):2575-2596, 2014.

[CW77] R. R. Coifman and G. Weiss. Extensions of Hardy spaces and their use in analysis. Bull. Amer. Math. Soc., 83(4):569-645, 1977.

[DH72] J. J. Duistermaat and L. Hörmander. Fourier integral operators. II. Acta Math., 128(34):183-269, 1972.

[DSFS14] D. Dos Santos Ferreira and W. Staubach. Global and local regularity of Fourier integral operators on weighted and unweighted spaces. Mem. Amer. Math. Soc., 229(1074):xiv+65, 2014.

[Dui96] J. J. Duistermaat. Fourier integral operators, volume 130 of Progress in Mathematics. Birkhäuser Boston, Inc., Boston, MA, 1996.

[Esk70] G. I. Ėskin. Degenerate elliptic pseudodifferential equations of principal type. Mat. Sb. (N.S.), 82(124):585-628, 1970.

[FS72] C. Fefferman and E. M. Stein. $H^{p}$ spaces of several variables. Acta Math., 129(3-4):137-193, 1972.

[Fuj79] D. Fujiwara. A construction of the fundamental solution for the Schrödinger equations. Proc. Japan Acad. Ser. A Math. Sci., 55(1):10-14, 1979.

[HLZ15] Q. Hong, G. Lu and L. Zhang. $L^{p}$ boundedness of rough bi-parameter Fourier integral operators. arXiv:1510.00986.

[Hör71] L. Hörmander. Fourier integral operators. I. Acta Math., 127(1-2):79-183, 1971.

[Kg76] H. Kumano-go. A calculus of Fourier integral operators on $R^{n}$ and the fundamental solution for an operator of hyperbolic type. Comm. Partial Differential Equations, 1(1):1-44, 1976.

[KgN70] H. Kumano-go and M. Nagase. $L^{p}$-theory of pseudo-differential operators. Proc. Japan Acad., 46:138-142, 1970.

[Miy80] A. Miyachi. On some estimates for the wave equation in $L^{p}$ and $H^{p}$. J. Fac. Sci. Univ. Tokyo Sect. IA Math., 27(2):331-354, 1980. 
[Per80] J. C. Peral. $L^{p}$-estimates for the wave equation. J. Funct. Anal., 36(1):114-145, 1980.

[RLS13] S. Rodríguez-López and W. Staubach. Estimates for rough Fourier integral and pseudodifferential operators and applications to the boundedness of multilinear operators. J. Funct. Anal., 264(10):2356-2385, 2013.

[RS06a] M. Ruzhansky and M. Sugimoto. Global $L^{2}$-boundedness theorems for a class of Fourier integral operators. Comm. Partial Differential Equations, 31(4-6):547-569, 2006.

[RS06b] M. Ruzhansky and M. Sugimoto. A smoothing property of Schrödinger equations in the critical case. Math. Ann., 335(3):645-673, 2006.

[RS11] M. Ruzhansky and M. Sugimoto. Weighted Sobolev $L^{2}$ estimates for a class of Fourier integral operators. Math. Nachr., 284(13):1715-1738, 2011.

[RS12a] M. Ruzhansky and M. Sugimoto. Smoothing properties of evolution equations via canonical transforms and comparison principle. Proc. Lond. Math. Soc. (3), 105(2):393-423, 2012.

[RS12b] M. Ruzhansky and M. Sugimoto. Structural resolvent estimates and derivative nonlinear Schrödinger equations. Comm. Math. Phys., 314(2):281-304, 2012.

[Ruz01] M. Ruzhansky. Regularity theory of Fourier integral operators with complex phases and singularities of affine fibrations, volume 131 of $C W I$ Tract. Stichting Mathematisch Centrum, Centrum voor Wiskunde en Informatica, Amsterdam, 2001.

[Ruz09] M. Ruzhansky. On local and global regularity of Fourier integral operators. In New developments in pseudo-differential operators, volume 189 of Oper. Theory Adv. Appl., pages 185-200. Birkhäuser, Basel, 2009.

[SSS91] A. Seeger, C. D. Sogge, and E. M. Stein. Regularity properties of Fourier integral operators. Ann. of Math. (2), 134(2):231-251, 1991.

[Ste70] E. M. Stein. Singular integrals and differentiability properties of functions. Princeton Mathematical Series, No. 30. Princeton University Press, Princeton, N.J., 1970.

[Ste93] E. M. Stein. Harmonic analysis: real-variable methods, orthogonality, and oscillatory integrals, volume 43 of Princeton Mathematical Series. Princeton University Press, Princeton, NJ, 1993. With the assistance of Timothy S. Murphy, Monographs in Harmonic Analysis, III.

[Sug92] M. Sugimoto. On some $L^{p}$-estimates for hyperbolic equations. Ark. Mat., 30(1):149-163, 1992.

[Tao04] T. Tao. The weak-type $(1,1)$ of Fourier integral operators of order $-(n-1) / 2$. J. Aust. Math. Soc., 76(1):1-21, 2004.

Michael Ruzhansky:

Department of Mathematics

IMPERIAL COLLEGE LONDON

180 QueEn's Gate, London SW7 2AZ, UK

E-mail address m.ruzhansky@imperial.ac.uk

Mitsuru Sugimoto:

Graduate School of Mathematics

NAGOYA UNIVERSITY

Furocho, Chikusa-Ku, Nagoya 464-8602, JaPAN

E-mail address sugimoto@math.nagoya-u.ac.jp 